INVESTIGACIÓN

http://dx.doi.org/10.15198/seeci.2017.43.115-133

Recibido: 24/03/2017 Aceptado: 15/04/2017

\title{
PARTICULARIDADES Y CONTRADICCIONES DEL CINE NORTEAMERICANO EN LOS INICIOS DEL PERIODO CLÁSICO
}

\section{PARTICULARITIES AND CONTRADICTIONS ON AMERICAN FILM INDUSTRY IN THE BEGINNINGS OF THE CLASSIC PERIOD}

José Hernández Rubio: Universidad Carlos III de Madrid. España hernarubio@hotmail.com

\section{RESUMEN}

Los inicios del concebido como periodo "clásico" del cine norteamericano también se vieron sometidos, al igual que el resto de ámbitos sociales y culturales, al formidable cambio que experimentaría Estados Unidos al encarar la década de los años treinta. En ese agitado contexto de retraimiento obligado, la Gran Depresión, la industria del cine se vio severamente afectada por la crisis socioeconómica; pero de sobra conocido es que también, en ese periodo, se estaban favoreciendo unos nuevos métodos y formas de hacer cine que iban a lograr un éxito descomunal, con enorme influencia en la producción del resto del mundo. Es precisamente la intención de este estudio indagar en las particularidades del primer cine clásico norteamericano, derivadas de abiertas contradicciones casi inexplicables, que no obstante tuvieron como respuesta el auge y consolidación del séptimo arte, llegando hasta nuestros días. Para dicho trabajo me he basado en los comentarios de varios críticos, economistas e historiadores de gran reconocimiento, así como de otros estudiosos menos conocidos. Igualmente, varias películas realizadas en aquellos momentos me han ilustrado certeramente sobre la realidad del país. Todos han aportado unas nociones específicas y muy valiosas que confirman el resultado final de grandiosidad e influencia de la industria del cine, a pesar de la coyuntura especial de Estados Unidos en la época.

PALABRAS CLAVE: Contradicciones - particularidades - inicios - clásico auge - películas.

\footnotetext{
${ }^{1}$ José Hernández Rubio: Doctor en Humanidades, Universidad Carlos III de Madrid. Profesor cursos de Cine y Comunicación Audiovisual en la Universidad del Mar (Universidad de Murcia). hernarubio@hotmail.com
} 


\section{ABSTRACT}

The beginning of American cinema known as "classic" period was also affected by the huge change that the United States would undergo at the dawning of the 1930s, which also affected social and cultural areas. In that hectic context of forced withdrawal, the Great Depression, the film industry was severely affected by the socio-economic crisis; but it is well known that in that period, new methods and forms of cinema were being favored, which were to achieve enormous success, with enormous influence on the production of the rest of the world. The intention of this study is precisely to research the particularities of the first North American classical cinema, which derived from open and almost inexplicable contradictions, which nevertheless resulted in the boom and consolidation of the seventh art, reaching even our days. For this paper, I have based myself on the comments of several well-known critics, economists and historians, as well as other lesser known scholars. Likewise, several films made at that time have clearly shown me about the reality of the country. All have contributed specific and very valuable notions that confirm the final result of grandeur and influence of the film industry, despite the special juncture of the United States at that time.

KEYWORDS: Contradictions-particularities-classic-early-rise- films.

\section{Cómo citar el artículo}

Hernández Rubio, J. Particularidades y contradicciones del cine norteamericano en los inicios del periodo clásico [Particularities and contradictions on american film industry in the beginnings of the classic period] Revista de comunicación de la SEECI, 43, 115-133. Doi: http://dx.doi.org/10.15198/seeci.2017.43.115-133 Recuperado de http://www.seeci.net/revista/index.php/seeci/article/view/467

\section{INTRODUCCIÓN}

Al abordar las diversas particularidades que conforman el primer cine norteamericano se debe establecer, aun con brevedad, la situación histórica que aconteció en Estados Unidos en la etapa de transición de los años veinte a los treinta.

Hay que señalar que en muy pocos meses pasaría de ser la máxima potencia mundial en materia económica, a través de sectores como el financiero y el bursátil, a convertirse en el mismo germen de una crisis socioeconómica sin precedentes, afectando irremisiblemente al resto de países, como bien es sabido. Por consiguiente, aquella prosperidad y crecimiento que se había instalado en la sociedad de los "felices años veinte", iba a derivar en carestía generalizada en todas las capas de la ciudadanía y de la América rural, a causa de la concatenación de varios factores, como el derrumbe de los sectores productivos, la mala gestión del gobierno Hoover, y sobre todo el crack bursátil 
de 1929 que abocó a la ruina a millones de norteamericanos. La Gran Depresión subsiguiente, sobradamente estudiada, provocó que la salida de la crisis fuese larga y traumática, con una pobreza extensiva por falta de expectativas de empleo, y con tan sólo el nuevo programa reformista del nuevo gobierno Roosevelt como hálito de esperanza. Pero al mismo tiempo que la mayor parte de los sectores productivos y de la propia industria se habían colapsado sin poder colocar sus productos, un fenómeno cultural y también industrial como el de la Cinematografía no sólo estaba consolidando su auge, sino que se iba a erigir en primera fuente de ingresos y de crecimiento. Por tanto, este trabajo pretenderá recoger una investigación de rigor, que pueda explicar esa gran paradoja que se suscitó en un país en estado de schock, testigo al mismo tiempo del fortalecimiento de un cine que facilitó el comienzo del periodo clásico.

\section{OBJETIVOS}

Se trata, por tanto, de analizar un conjunto de particularidades, incluso contradicciones, que a pesar de haber sido estudiadas exhaustivamente y por separado desde unos datos incuestionables, no impide que se expongan ahora de manera conjunta para lograr el objetivo principal. Será desde una perspectiva integradora, pues, donde se asiente la aportación de este estudio, para poner de relieve, por tanto, el porqué de esa inmejorable y paradójica oportunidad que tuvo el cine.

Sabemos que el "sueño americano" pronto iba a tocar a su fin con la eclosión de la bolsa de Nueva York a finales de 1929, con terribles consecuencias socioeconómicas en todos los ámbitos, incluso a nivel internacional, durante los siguientes años. Con todo, en esa larga etapa de "Depresión" estriba la primera contradicción, que habrá que analizar en lo referido a los inicios del apogeo de la industria del cine.

Por otro lado, se produjo una segunda curiosidad muy llamativa que estaba condicionando la razón de existir del mismo cine: la masiva asistencia a las salas por parte de una sociedad empobrecida. Por tanto, otro objetivo crucial será examinar las causas de la asistencia de una ciudadanía fiel al espectáculo, a pesar de la carestía general.

Como también hay que analizar la importancia de la renovación y cristalización de los géneros cinematográficos, que crecían con fortuna en un contexto de crisis en la misma industria del cine, que parecía salir airosa comparada con el resto de sectores. Además, otra contradicción palmaria fue el desarrollo dichoso de ciertos géneros de relevancia, como el cine negro o el social, frente al trabajo de una censura vigilante, a menudo agobiante para los creadores.

Otra particularidad no menor fue la respuesta de las fuerzas sindicales implicadas en la industria de la cinematografía, que optaron por un escenario 
de colaboración en lugar de la tradicional protesta. Por ello, el objetivo debe basarse en averiguar las causas del cambio de postura.

Gracias a la afortunada conjunción de los factores anteriores, junto a las nuevas formas de producir que imponía el llamado Sistema de Estudios, y al descubrimiento de nuevas técnicas sonoras y fímicas, se establecían los inicios de la etapa clásica del cine norteamericano.

\section{METODOLOGÍA}

Para abordar este epígrafe, hay que citar los diversos recursos metodológicos empleados, que han permitido esclarecer cada una de las particularidades aludidas.

En primer lugar, en lo referente al contexto estrictamente histórico de la Gran Depresión, hay que dejar constancia de cierta terminología social y política suscitada en la época; a su vez, son definiciones que están muy relacionadas con la evolución económica de los sectores productivos, y por supuesto, con el de la industria del cine. Así, al hablar de "la diplomacia del dólar", acepción atribuida al presidente Theodore Roosevelt y generalizada entre los economistas de las primeras décadas del siglo, se aludía a la capacidad de influencia económica totalizadora de Estados Unidos en otros países. En concreto, se refería a las iniciativas norteamericanas de gestionar préstamos, deudas de guerra, aranceles y estabilización monetaria, para reforzar sus intereses en el exterior.

Por otro lado, otra noción latente es la del archiconocido "sueño americano", como consigna para un joven país pleno de expectativas de vida. En su libro The Epic of America (La Épica de América) publicado en 1931, el historiador norteamericano James Truslow Adams reflexionaba sobre las bases del American Dream:

Ese sueño de una tierra en la que la vida debe ser mejor y más rica y más llena para todos, con oportunidades para cada uno de acuerdo a sus habilidades y hazañas. No es simplemente un sueño de coches y altos salarios, sino un sueño de un orden social en el que a cada hombre y a cada mujer debe permitírsele alcanzar la más alta cota de la que sean capaces innatamente, y ser reconocidos por otros por aquello que son, independientemente de las circunstancias fortuitas de su origen 0 posición (Truslow, 1931).

Recordemos también la prohibicionista y contraproducente Ley Volstead, apenas cumplida con rigor dado el clima de laxitud con el alcohol en la época, aunque se confiscasen miles de destilerías ilegales. Se trata de una ley que afectaba muy negativamente a la ciudadanía de las grandes urbes, sobre todo. $Y$ tiene mucho que ver con un clima inaguantable de inestabilidad social, además de lo puramente económico, como también, con la inspiración de un género cinematográfico de enorme éxito, como el del "gangsterismo". Por su 
parte, otro concepto crucial es el del mismo crack, que como dice el economista Joseph Galbraith, se refiere a:

El demencial alejamiento de la razón: detrás del auge de la especulación estaban las distintas fuerzas -económicas, culturales, psicológicas y políticas. No puede, en efecto, caber ninguna duda razonable respecto del hecho de que el hundimiento de los valores bursátiles, el desplome de las fortunas de aquellos que jugaban en el mercado, y el gasto del consumidor al igual que para las empresas, tuvieron una influencia traumática sobre la producción, el ingreso y el empleo. (Galbraith, 2000, p.15).

Y como es bien sabido, el programa de reformas para intentar sacar adelante a Estados Unidos se instauró en el "Nuevo Trato" o New Deal, del gobierno Franklin D. Roosevelt. Tuvo mucho que ver con la recuperación, a lo que habría que añadir que bajo su paraguas político el cine se vio ampliamente difundido, es más, ambos se vieron mutuamente favorecidos.

En cuanto a la metodología para investigar las características especiales de la industria del cine en sí, este trabajo ha ido encaminado a rescatar diversas ideas esclarecedoras de expertos de gran reconocimiento, en la dirección adecuada de sintetizar las conclusiones que se persiguen. Entre otros, los historiadores norteamericanos más relevantes son Janet Staiger, colaboradora inestimable de la Historia General del Cine de la editorial Cátedra, en su volumen VI (1995), donde facilita datos numéricos precisos sobre recaudación y asistencia del público a las salas; o Giuliana Muscio en la Historia Mundial del Cine de la editorial Akal (2011), donde explica los pormenores de las huelgas de escritores y guionistas ante la rigidez de un studio-system que coartaba la creatividad de aquellos; o David Bordwell y Kristin Thompson que con sus aportaciones en el manual de referencia indiscutible, The Classical Hollywood Cinema: Film Style and Mode of Production to 1960 de la editorial Paidós (5a ed. 1997) ofrecen unas reflexiones de gran valía que profundizan en la capacidad del ámbito financiero y bursatil (JP Morgan Chase, National City Bank, Bolsa de Nueva York...) para influir en las decisiones de las compañías cinematográficas; o Douglas Gomery, que en la Teoría y práctica de la historia del cine, también de la editorial Paidós (1985) ha indagado en la dinámica de gestión administrativa y económica de las propias Majors.

Así mismo, el enfoque aportado por otros escritores e historiadores del cine españoles, se ha situado en la misma línea de exponer dichas peculiaridades. En este sentido, la metodología se ha asentado en recoger ciertos artículos muy notables e ilustrativos. Así, en el número 116 de la revista Dirigido por, el artículo "Hollywood en silencio. La llegada del sonoro" (1994), José Enrique Monterde ha examinado la repercusión del sistema sonoro en la estructura cinematográfica de aquella época, donde se iba a institucionalizar un método revolucionario y casi definitivo, merced al papel de los grandes estudios en los costosos gastos de reconversión. Además, Carmen Mainer, con el estudio "El cine norteamericano durante la Gran Depresión (1929-1939)" en la revista Fotocinema número 6 de 2013, profundiza en la política cultural de la era 
Roosevelt y su apoyo decidido a cineastas como Frank Capra o King Vidor, que al mismo tiempo que filmaban sin ambages los estragos de la crisis, eran capaces de insuflar esperanza y autoestima en obras memorables. Y más específicamente, Miguel Marías con "William Wellman, un cineasta misterioso" publicado en el número 146 de la Revista Filmoteca Generalitat Valenciana (1994), aborda la trascendencia de un director emblemático que, desde una tipología de cine social supo acometer la cara menos amable de Estados Unidos en la década de los treinta, muy lejos de las edulcoradas y evasivas producciones que Hollywood.

Como no podía ser menos, otro procedimiento imprescindible ha sido el análisis breve de determinados filmes, que gracias a su calidad artística ya se han convertido en propiamente clásicos, de merecido reconocimiento cultural. Y también implican unos modelos de gran calado para un vasto número de películas realizadas en las décadas siguientes, independientemente de su excelencia. Con ellos, se atestigua la variedad cinematográfica que estaba germinando en los años treinta (con muchas raíces anteriores), con nuevos géneros y enfoques, desde la finalidad del puro entretenimiento hasta la búsqueda de reflexión ciudadana.

Considerando que esta metodología ha resultado suficiente y útil, también hay que mencionar los recursos del resto de referencias bibliográficas, o bien, otras obras cinematográficas que de manera similar hubieran contribuido con solvencia al resultado final.

\section{DISCUSION}

Para ahondar en esa idea de gran paradoja en el cine de la Depresión, se ha de recurrir a las circunstancias ya esbozadas, como explicación fehaciente y objetiva de dicha coyuntura tan especial.

\subsection{Un agitado contexto histórico}

En primer lugar, para explicar las circunstancias del soporte histórico, ya esbozado, de un país como Estados Unidos, hay que retrotraerse a los primeros factores de incoherencia sociopolítica a finales de los años veinte: menosprecio a la Sociedad de Naciones, aranceles cortoplacistas, no estimulación de un programa económico de calado, ni previsión de crecimiento de desempleo, liberalismo desregulado a ultranza, o especulación brutal en la Bolsa, donde los bancos prestaban a la ciudadanía a un interés alrededor del $12 \%$, para colocarlos en acciones a veces al 50\%. Y habría que sumar el frágil sistema bancario: miles de bancos regionales e independientes con unos activos muy limitados. Los historiadores han constatado sobradamente que al cambiar con rapidez la Bolsa, fruto de la máxima tensión especulativa en octubre de 1929, miles de ahorradores comenzaron a liquidar sus pertenencias, acuciados por el pánico: más de 13 millones de títulos que cotizaban a la baja no encontraron compradores. Las consecuencias del caos económico-financiero no se hicieron 
esperar y Estados Unidos comenzaba una década muy distinta a la anterior: cierre masivo de empresas industriales y agrícolas, descenso del consumo, acumulación de excedentes, quiebra de más de 600 bancos sin reaseguro de la Reserva Federal, impagos millonarios, etc.

Paralelamente, la ciudadanía norteamericana se estaba sometiendo peligrosamente a una creciente decadencia, luego de la época dorada de los años veinte: corrupción entre las altas esferas políticas y empresariales (amigos del mismo presidente Harding: la llamada "Banda de Ohio"), complicidades entre gangsterismo, políticos, policías y jueces, (ascenso de personajes como Al Capone), poco ejemplar Ley Volstead, en "virtud" de la cuál ciertas élites sociales promovían fiestas regadas de alcohol en sus mansiones, fenómenos migratorios de segunda generación que tenía en el crimen organizado su salida más fácil, progresiva brecha entre las clases sociales, arrinconando a millones de habitantes parados de toda Norteamérica a los extrarradios urbanos hacinados y míseros... En definitiva, se materializaron de manera contundente aquellos factores adversos que estaban vaticinando la crisis. Constatemos además, los movimientos de población en busca de trabajo (de 4 millones de parados en abril de 1930 se pasó a 15 millones en julio de 1932), o los "salarios de hambre". Una auténtica angustia generalizada sacudiría al país durante un largo periodo, y como dice, Pedro Schwartz:

Suele hablarse de tres años de crisis en EEUU, los que van de 1929 a 1933, cuando en realidad fueron diez. Para el país, la década de 1930 fue toda una década perdida. Si a lo largo de sólo once meses entre 1930 y 1931, los últimos años de Hoover, la cantidad de dinero en la economía americana se redujo en un $26 \%$, hoy se acepta que esa repentina desaparición de los recursos de pago agravó la recesión y la prolongó innecesariamente. (Schwartz, 2009, p.455).

El malestar colectivo se reflejó abiertamente en los resultados de las elecciones presidenciales de noviembre de 1932, al otorgar la confianza al demócrata Franklin D. Roosevelt y su esperanzador programa reformista del "Nuevo Trato" o New Deal, como bien sabemos. En el plano internacional, la crisis provocó un mayor empobrecimiento de las arcas del viejo continente que observaba cómo sus stocks no tenían salida.

\subsection{Auge de la industria del cine en un contexto socioeconómico adverso.}

Irremediablemente, la transformación histórica tan dura con los acontecimientos anteriores afectó a la evolución de la industria del cine. En efecto, la influencia de la crisis en las estructuras de un arte tan joven lo abocaba a la pronta desaparición. Como ha estudiado Galbraith (2000), el cine se resintió de tal manera que muchas empresas medianas tuvieron que desaparecer, fueron incapaces de asumir los costes de producción por falta de circulación económica, de desconfianza de los inversionistas y de otras empresas publicitarias. 
Con todo, de manera paradójica, en un escenario histórico tan adverso, la consolidación del cine en sus comienzos del clásico fue un hecho incontestable. En primer lugar se ha de advertir que su supervivencia residió, en buena medida, en que fue el sector que más tardó en sufrir las consecuencias del caos económico, con lo que el afianzamiento progresivo de Hollywood parecía estar convirtiéndose en una constante, sobre todo desde la llegada del cambio político. El programa de reformas que implicaba el New Deal (Nuevo Contrato) se iba a convertir en un compañero inestimable para el cine. Al respecto, como sintetiza Carmen Mainer (2013, pp.171-200), ya como gobernador del estado de Nueva York, Roosevelt había llevado a cabo en los primeros años de la crisis un paquete de reformas socioeconómicas destinadas a superarla, intentando frenar el descontrolado liberalismo económico sin reglas; de todo ello se infiere que pese a los estragos de la crisis, una inmejorable oportunidad de recuperación empezaba a generarse en diversos sectores, en especial en el de la cinematografía, muy apreciado por las estructuras del poder político, por su potencial económico y sociológico. Sin embargo, también hay que apuntar que la fortaleza de las Majors de Hollywood no se hizo efectiva hasta mediados de los treinta, después de muchos vaivenes, proyectos fallidos, suspensiones de pagos frecuentes, teniendo que optar por la solución menos lesiva: la absorción o la fusión entre las menos potentes.

Otra singularidad de la no desaparición total del cine se asentaba en la ironía de que, algunos de los factores que afectaron negativamente a la industria serían los que también intervendrían en la ralentización de su hipotético hundimiento, incluso en su renovación poco después. Se ha verificado suficientemente cómo, en un primer momento, la industria del cine quedó seriamente tocada por las decisiones de grandes empresarios, que provocaron una primera caída de la asistencia: según los datos facilitados por la Standard Trade and Securities, recogidos por Janet Staiger (1995, p.143), se pasó de 1.100 millones de dólares de recaudación en 1929 a 625 millones en 1932, e igualmente, los recortes de personal en los diversos oficios apegados al séptimo arte afectaron por igual a directores, intérpretes u operarios, e incluso a la desaparición de empresas subsidiarias de maquillaje, vestuario, decorados, etc. Pero frente al declive, resurgió la apuesta decidida de aquellos mismos responsables que, desde el riesgo económico palmario siguieron reestructurando el negocio con vehemencia. Al respecto, la industria del cine había estado muy ligada al mundo de las finanzas, en manos de unos productores que también habían participado en la especulación bursatil, y que en la primera etapa de la Depresión todavía se dispusieron a apoyar iniciativas rentables, desde su conexión con Wall Street. Como resume George Sadoul: "La gran crisis que abrió la quiebra de la Bolsa de Nueva York tuvo por consecuencia una renovación -con frecuencia fecunda- de los creadores, a la vez que un esfuerzo del dominio de Wall Street sobre el arte y la industria del film" (Sadoul, 1991, p.205). Una tesis similar es la de David Bordwell (1997, p. 350): el hecho de que ciertos valores bursátiles del sector cinematográfico tuvieran que soportar los vaivenes propios de la inestabilidad, no impidió que las altas esferas cercanas a los Morgan y Rockefeller controlaran, mediante fuertes sumas de 
dinero, la adaptación a los nuevos equipamientos del sonoro, dominio de patentes o imposición de ejecutivos clave en las juntas directivas. Así mismo, Bordwell comenta la importante diferenciación entre "el interés de los directivos por el crecimiento global de la empresa, en vez de conseguir unos dividendos elevados a corto plazo, práctica habitual de los meros propietarios" (Bordwell et al, 1997, pp.352.353).

Quedaba así reforzado el cambio estructural donde los economistas controlaban todo el proceso de trabajo y casi el diseño del producto cinematográfico, bajo una supervisión central en la gestión mucho más dura que antes, lo que acarreaba otra gran contradicción referida al trabajo artístico de cineastas y guionistas, en definitiva a la esencia cultural del cine. Obviamente, a la altura de los primeros años treinta, los máximos implicados en defender el sector desde cualquier perspectiva, económica o cultural, no eran conscientes de que comenzaba un periodo largamente fructífero y universal, para servir de modelo artístico para la creatividad posterior. Bajo ese sistema integral de producción auspiciado por Hollywood, para trabajar con la máxima coordinación entre sus partes, se lograron unos frutos que han pasado a la historia del cine como sobresalientes y eternos,.... cuyo origen partió de una situación de crisis galopante.

\subsection{Consolidación del Sistema de Estudios frente a la crisis cinematográfica.}

Grandes compañías como la Columbia, la Fox y la Paramount habían comenzado a recurrir, desde finales de 1931, a un sistema de producción con gran éxito económico, consistente en regular y administrar la tirada y especializar a las compañías en determinados géneros. Por el contrario, se forjaba una desprotección y una precariedad que afectaban a las más modestas, por parte de la misma administración y de los inversores privados. La seguridad de las grandes radicaba en el control de un auténtico oligopolio capitalista. Los procesos de concentración, pues, constituía la premisa mercantil fundamental para poder hacer películas de enorme presupuesto y aceptación para un público masivo. El recién concebido como Studio System era el protagonista de la hegemonía de la industria del cine norteamericano. Acaparar en más de un $90 \%$ tal sector significaba que apenas se podía entrar a competir, lo cual estaba forjando el carácter definitivo de un Hollywood cuasi totalitario.

Frente a las limitaciones de la economía nacional, una inicua industria como la del cine triunfaba según su jerarquizado sistema de organización, lo que no dejaba de resultar llamativo. En las dificultades de la Gran Depresión, incluso para muchos sectores del séptimo arte, el crecimiento de las grandes compañías floreció sin cesar. Además, mantuvieron a raya las disposiciones del gobierno federal durante décadas, enfrentándose a algunas infracciones antimonopolistas que pretendían frenar la acaparadora explotación de la industria, como la National Industrial Recovery Act (Ley de Recuperación de la Industria Nacional), que finalmente obtuvo magros resultados, ya que el mismo Estado necesitaba del tirón empresarial para relanzar la economía. El resultado 
real de los pleitos derivaba, por tanto, en otra particularidad: el control de la industria por parte de los grandes llegó a tales extremos porque que el beneficio también repercutía en el mismo gobierno demócrata. Los newdealers no querían considerar el trust de suficiente envergadura como para determinar un monopolio estricto.

Por otro lado, otra singularidad bien contrastada, nos habla de que la fuente de poder de las majors residía en la extensa red de distribución que poseían en el propio país y en el mundo, antes que en un número desorbitado de producción de películas. Como industria próspera, el cine se preocupó de seguir produciendo en abundancia en un entorno de depresión socioeconómica, con películas de toda calidad y género, pero su verdadero auge obedecía a la capacidad de llegar a cualquier rincón del planeta. Al respecto, Douglas Gomery sintetiza que, "El poder de las cinco grandes compañías y las tres pequeñas en el sector de la distribución, suponía que la recaudación procedente del extranjero fuese aproximadamente la mitad de los ingresos de un largometraje medio" (Allen y Gomery, 1985, p.33).

\subsection{La respuesta sindical de colaboración.}

En principio, la respuesta sindical a la gestión de Hollywood, máxime con el advenimiento del cine sonoro, acrecentó la tensión en todos los perfiles profesionales. Las reestructuraciones laborales se dejaron sentir de manera grave, desde los trabajadores de a pie a los mismos autores cinematográficos. La historia del cine nos dice que numerosos técnicos, guionistas y directores, agrupados en los llamados sindicatos creativos como el Screen Writers Guild o el Screen Directors Guild, "estuvieron muy afectados por la deriva económica y los problemas sociales, así como del sentido de frustración ante la primacía del rígido y jerárquico studio-system y de la cuestión comercial" (Muscio, 2011, p.503). Y las primeras presiones obreras se dejaron sentir tras octubre de 1929: fueron intensos los conflictos de trabajadores con el Warner, cuya faceta menos grata quedó en evidencia al despedir a 900 empleados y bajar los salarios al resto.

En cambio, aquejado por las circunstancias, el poder sindical se iba a convertir más tarde en oportunidad de colaboración. En efecto, los conflictos en la negociación laboral no impidieron que se forjase una especialización del trabajo en época de crisis. Es decir, que para hacer frente al lamentable estado socioeconómico, "las prácticas de sindicación no se enfrentaron tanto al sistema de producción como al afianzamiento de la subdivisión del trabajo" (Bordwell, et al, 1997, p.348). Incluso se produjeron enfrentamientos entre los mismos sindicatos por la disputa de ciertas labores, que el Estado hubo de solventar. De todo ello se desprende otra aparente contradicción: del teórico y eterno choque entre empresas y sindicatos se iba a pasar a un sistema de equilibrio forzoso. Frente a la tradicional postura de protesta de los trabajadores de base, se transitó con frecuencia a una suerte de colaboración como mal menor. Y a pesar de ciertos vaivenes en la pugna laboral, se deseaba que las compañías 
pudiesen desarrollar sus cometidos en aras de no perder puestos de trabajo. Se trataba de una actitud que el mismo cine representó:

"La creciente fuerza sindical significó una evolución en el tratamiento de la figura del obrero en las películas realizadas en Hollywood entre 1932 y 1940, pasando de referir las acciones de protesta obreras como fruto del resentimiento o la ambición, a entender a la clase obrera organizada como una fuerza positiva a implicar en la empresa de reconstrucción nacional" (Monterde, 1997, p. 138).

\subsection{Pobreza social versus masiva asistencia a las salas.}

Por lo que respecta a la asistencia de la ciudadanía al cine de la "poscrisis", tenemos varias versiones que corroboran otra gran paradoja: el masivo seguimiento popular. Ángel Luis Hueso (1988) nos ilustra sobre lo llamativo que resultaba la fidelidad del espectador entregado a sus ídolos en la pantalla (además de escapar de la frustración cotidiana que infligía el malestar socioeconómico), o la muy apreciada novedad del recién descubierto formato sonoro, o el gusto colectivo por los nuevos y diversos géneros. Por su parte, Edward Epstein nos dice que "ninguna forma de entretenimiento producida centralmente había cautivado jamás una proporción tan numerosa de población" (Epstein, 2007, p.331). En cambio, muy otra es la opinión de Guliana Muscio, que apunta a que se ha de matizar mucho sobre la asistencia masiva de aquella sociedad devota del cine, tan igualada por la crisis, ya que era una asistencia que el mismo gobierno fomentaba, incluso con el cambio político:

Durante la Gran Depresión todo el mundo frecuentaba las salas. El cine fue una necesidad para la administración Hoover, la cual, en plena crisis de los primeros años distribuía comida, ropas y entradas para las salas; entradas para alejar a la gente de la calle, ofrecerle refugio y consuelo; si queremos decirlo así: 'panem et circenses' (Muscio, 1996, p.25).

Incluso, como sobradamente se ha estudiado, las propias salas de exhibición hacían sorteos y distintos regalos entre el público. Y en efecto, la respuesta política y social a una crisis de tal calibre, sobrevenida e imprevisible para quienes dirigían el destino del país, era no alarmar con augurios negativos todavía más a sus súbditos. Se trataba de ofrecerles productos de evasión, de simpleza moral frente a la dura y compleja cotidianeidad (de nuevo otra ironía): la mayor parte de las veces las películas de Hollywood estaban atadas a sus definitivas narraciones convencionales.

Which of them were the result of the structure of big studio commerce. As such, the movies expressed a fairly consistent aesthetic and ideological viewpoint. The complex and often contradictory nature of certain issues were reduced to an easily contained dramatic conflict between good and evil forces, necessarily resolved in the Happy Ending. With few exceptions, such oversimplification of social problems tended to provide a rather reactionary political message (Roffman y Purdy, 1981, p.8). 
Cualquiera de ellas era el resultado de la estructura de un gran estudio comercial. De tal forma, las películas expresaban una aceptable constante estética y un punto de vista ideológico. El complejo y con frecuencia contradictoria naturaleza de ciertas cuestiones eran reducidas a un accesible conflicto contenido dramático entre fuerzas buenas y malas, necesariamente resueltas con el Final Feliz. Con pocas excepciones, tal simplificación excesiva de los problemas sociales tendía a proveer un contundente mensaje político conservador.

Se ha de señalar además, a "esa vasta clase media norteamericana que todavía disponía de expedientes y recursos que de ninguna manera eran tan abundantes en otros países" (Lacolla, 2008, p. 165), por lo que es fácil intuir que a pesar de verse reducido el número de espectadores en un tercio entre 1930 y 1933 , la gente no quería prescindir de un entretenimiento relativamente barato. La conclusión general nos debe llevar a que, bien por idolatría hacia el cine y su mundo, bien por el apoyo del gobierno o porque las compañías facilitaban el acceso a las salas, la asistencia del espectador norteamericano no cayó en demasía. En este sentido, resulta muy esclarecedor recordar que el hecho de "ir al cine" no implicaba per se "ver una película". El entretenimiento consistía en una suerte de paquete de ocio cultural que incluía un espectáculo teatral, un noticiario, varios cortometrajes y la película anunciada, como años atrás, donde "era muy posible que para muchos de los clientes no tuviese demasiada importancia qué película se proyectaba" (Allen y Gomery, 1985, p.203).

Por último se ha de recordar que con la aparición de los premios Oscars, desde su primer edición de 1929, la afluencia del público se incrementaría sobremanera en los años siguientes.

\subsection{Cristalización de unos géneros escapistas a pesar de la censura.}

Ya se ha comprobado cómo aquellos preámbulos del cine clásico estuvieron sujetos a unas disciplinas productoras, que junto al protagonismo de las nuevas técnicas del sonoro, implicaba un fortalecimiento de nuevas formas de expresión. $Y$ "aquellas empresas que no entendieron que había que apostar por fuertes inversiones en artefactos e infraestructuras, ya que así lo exigía el cine sonoro, desaparecieron o se vieron relegadas en el mercado cinematográfico" (Monterde, 1994, p.17). Por tanto, la particularidad radica en que, en esta década van a nacer o madurar formas nuevas, unos géneros cinematográficos que un público voraz consumiría gracias a la atracción que suponía la escucha de los diálogos o de la música. Como nos recuerda Monterde:

La irrupción del cine sonoro completó la institucionalización de un modo de representación dominante en todo el mundo. Este propulsó el definitivo predominio de las grandes corporaciones, que eran las únicas en condiciones de afrontar los costosos gastos de reconversión de los estudios de rodaje (1997, p.132). 
Por otro lado, gracias a los géneros, el público sabía y conocía lo que iba a ver y el productor daba al público lo que éste esperaba que le diesen, a pesar de las limitaciones impuestas por la censura. En este sentido, conviene señalar que antes de la censura oficial de 1934, la Motion Picture Producers and Distributors of America (MPPDA) con el puritano Will Hays a la cabeza, como respuesta a la indignación pública ante los escándalos de Hollywood, ya había estado aplicando al cine unas reglas muy estrictas de moralidad tras el inicio de la crisis de 1929. Algunos sectores de la sociedad opinaban que los "excesos" hollywoodienses habían estado impregnando el imaginario colectivo, por lo que "se instauró un orden moral y, con él, una visión frecuentemente hipócrita que tendía a ignorar algunas de las realidades más fundamentales de la vida" (Coursodon, 1996, p.231). La contradicción, en este sentido, debe centrarse en que mientras lo estipulado en los acuerdos entre la industria, los grupos de presión y el mundo financiero ordenaba una vigilancia estrecha en las películas, algunos géneros florecieron precisamente gracias a realizaciones concretas que sorteaban las prohibiciones. Observemos someramente algunos géneros prominentes de los años treinta, con algunas películas susceptibles de control, o bien, destinadas al escapismo y evasión fomentado en la Gran Depresión.

Del cine cómico silente asentado en la pantomima y en la gestualidad, se pasó a la comicidad de exceso verbal que entrañaba el screwball comedy, de delirantes diálogos entre líos y desencuentros de parejas. Se llegaría así a una adhesión del público que valoraba un tipo de cine de humor muy refinado. Se trataba de crear situaciones estrafalarias con una planificación muy cuidada, donde a menudo se esquivaba las directrices del equipo censor, máxime con la figura de la mujer en momentos algo comprometidos. Directores tan importantes como Frank Capra o Ernst Lubitsch, y actrices como Katharine Hepburn o Myrna Loy encandilaban al espectador. Al servicio de las damas (My man Godfrey, Gregory LaCava 1934), Sucedió una noche (It happened one night, Frank Capra, 1934), o La fiera de mi niña (Bringing up baby, Howard Hawks, 1938), fueron muy reconocidas, apostando por una evasión consciente en el espectador. Pero además, otra curiosidad a destacar fue la del sentido de fantasía y lujo que destilaban ciertas películas con personajes adinerados... para un público paupérrimo las más de las veces. Así, Stanley Cavell ya explicaba:

There are comedies of the period which might better fit the description fairy tales for the Depression. But this seems less a reflection of particular economic realities or fantasies, than of the ancient theme of fairy tales of having wishes granted, call this the fantasy of escaping the realm of economy altogether... We honor the rich because they have externally the freedom, power, and grace which we feel to be proper to us (1999, pp.5-7).

Hay comedias de este periodo que responderían mejor a la descripción de cuentos de hadas para la Depresión. Pero ello no parece tanto un reflejo de fantasías o realidades económicas particulares, como del tema ancestral de los cuentos de hadas para que se cumplan los deseos, llamémosle la fantasía de escapar por completo del ámbito de la situación economía... Reverenciamos a los ricos porque, externamente, tienen la 
libertad, el poder y la elegancia que consideramos propia de nosotros mismos.

Por otro lado, el género musical también formaba parte de esta contraposición entre un mundo de glamour y un público arrobado y pobre. Su plenitud está ampliamente constatada, y el hecho de que en los primeros años del sonoro se contemplara una superproducción de musicales mediocres, no fue impedimento para que se asistiera a una renovación muy receptiva. Surgieron obras muy notables, con actores y actrices que colmaban los sueños del público, escapando momentáneamente de la dura realidad merced a los fabulosos números de música y coreografía, como en La calle 42 (42 nd street, Lloyd Bacon, 1933). Al respecto, hay que citar a la pareja formada por Ginger Rogers y Fred Astaire en memorables piezas musicales, siempre en estado de gracia, en películas como Sombrero de copa (Top hat, Mark Sandrich 1935). Por su parte, una joven actriz sobresaliente como Judy Garland, con Melodías de Broadway (Broadway Melody, Roy Del Ruth, 1937) marcaría otro hito.

También fue muy prolífico el western de los años treinta con la intención del puro divertimento, con el incipiente sonoro en marcha. La pura acción, las persecuciones, los peligros y enfrentamientos, el bien y el mal, la mitología y la épica, eran parámetros bien definidos que colmaban el ocio de unos ciudadanos deseosos de apartar las penurias cotidianas. Algunos westerns muy apreciados fueron La gran jornada (The big trail, Raoul Walsh, 1930), Billy el chico (Billy the kid, King Vidor, 1930) y Cimarron (Cimarron, Wesley Ruggles 1931.

Otra creación del sonoro fue el cine norteamericano de terror, que en definitiva albergaba la misma particularidad que el resto de géneros. Se progresó técnicamente en el miedo que producían los sonidos lúgubres, y las compañías cinematográficas se encargarían de explotar y rentabilizar a todo el elenco prototípico de monstruos, vampiros y zombis para morbo del espectador, pero bajo la supervisión censora. Y tras el éxito de Dracula (Dracula, Tod Browing, 1930) o de El doctor Frankestein (Frankestein, James Whale, 1931), el cine de terror se constituyó en una novedad cinematográfica muy visitada, pese a la carestía social. Dos años después, se estrenarían con gran éxito El hombre y el monstruo (Dr. Jekyll y Mr. Hyde, Rouben Mamoulian, 1932) y La parada de los monstruos (Freaks, Tom Browing, 1932).

La censura también se encargaría de poner cortapisas a ciertos filmes o secuencias cinematográficas de dos categorías ciertamente escabrosas, que mostraban sin tapujos la realidad lamentable de Estados Unidos: el cine de temática social y el de gangsters, todavía con el gobierno Hoover. "Pero la existencia de la censura no minimiza el valor del cine como testimonio sino que, incluso, hasta lo aumenta, pues como cualquier otra fuente, el cine puede ser tan revelador por lo que dice explícitamente como por lo que no dice o no se le permite decir" (Ibars R. y López I., 2006, p.11). En dichas categorías se recreaba una perspectiva comprometida con los verdaderos problemas de un país en estado de shock. Con todo, hasta que Roosevelt no accedió a la 
presidencia a finales de 1932, se estaba suscitando una gran particularidad en el primer gobierno de la Depresión, pues si por un lado era proclive a enmascarar la penosa realidad social, por otro no podía frenar el documentalismo cinematográfico de realizadores no profesionales, muy alertas con lo que acontecía a diario.

The Hoover Administration consistently minimized the suffering and hardship brought on by the economic crisis; the commercial cinema followed suit. Outraged by such neglect, amateur filmmakers throughout the country sought to document social conditions; supported by funds from the Workers International Relief, Film and Photo League branches were formed in Philadelphia, Detroit, Chicago, Los Angeles, San Francisco, and New York. It completed a series of short films and silent motion pictures on the hungry, the unemployed, the demonstrations, the breadlines, the evictions and others in their daily existence and activities (Davidson, 1993, pp.69-88).

La Administración Hoover minimizó constantemente los sufrimientos y las dificultades provocada por la crisis económica; el cine comercial hizo lo mismo. Indignado por esas negligencias, cineastas aficionados de todo el país trataron de documentar las condiciones sociales; con el apoyo de los fondos de los Trabajadores de Socorro Internacional, escuelas de la Liga del Cine y la Fotografía se formaron en Filadelfia, Detroit, Chicago, Los Angeles, San Francisco y Nueva York. Se completó una serie de cortometrajes y secuencias mudas sobre el hambre, el desempleo, las manifestaciones, las colas del pan, los desalojos y otros muchos en su vida y actividades de todos los días.

Sin embargo, algunas películas sí vieron la luz gracias a su más que merecido reconocimiento artístico y a la preocupación social que destilaban. Por ejemplo, una temprana Mendigos de la vida (Beggars of life, 1928) del citado William Wellman, ya estaba mostrando la desgraciada situación de ciertas capas de ciudadanos, anticipando de alguna manera el desastre brutal. En este sentido, "Wellman prefería ser franco y hosco en sus películas, que son provocativas, desafiantes y expeditivas" (Marías, 1994, p.23). Como también, en plena crisis, El pan nuestro de cada día (Our daily bread, King Vidor, 1934) o Los viajes de Sullivan (Sullivan 's Travels, Preston Sturges, 1939) suponían un retrato feroz y auténtico de una Estados Unidos desahuciado. En cuanto al género de gangsters, cabe destacar por su violencia Scarface, el terror del hampa (Scarface, Howard Hawks, 1932) y Soy un fugitivo (I Am a Fugitive From a Chain Gang, Mervyn Le Roy, 1933), donde se denunciaba las lamentables condiciones de los presos, algo no muy del gusto de la censura del Código Hays. La esencia de todas ellas consistía, al mismo tiempo, en su carácter lúdico y en concienciar al espectador, paradójicamente.

\section{CONCLUSIONES}


Las nociones que se han expuesto en este artículo están intrínsecamente relacionadas con la intención propuesta en la introducción, los objetivos y la discusión. Por tanto, la síntesis primordial que debe permanecer es la constancia de unas particularidades que favorecieron la consolidación, esplendor e influencia mundial del primer cine clásico norteamericano. Dicha conclusión se asienta principalmente en que, pese a un difícil contexto socioeconómico sin visos de recuperación, una industria cultural y comercial de enorme impacto estaba iniciando una época dorada. Para ello, de cada uno de los epígrafes anteriores se despejan unos resultados que conforman, de manera conjunta, esa gran paradoja de la grandiosidad del cine en plena Gran Depresión de Estados Unidos. De manera concisa, recordemos dichos rasgos específicos, a menudo contradictorios:

1. La crisis afectó a la industria del cine, pero lejos de declinar como el resto de sectores, fue reimpulsada por ciertas élites empresariales, financieras y políticas.

2. La gestión economicista del Sistema de Estudios de Hollywood permitió aliviar la producción, a veces en detrimento de la creatividad autoral.

3. De manera insólita, una sociedad empobrecida siguió acudiendo a las salas, pese a unos primeros años de caída de recaudación.

4. Se engendraron unos modelos cinematográficos, algunos preexistentes, para la realización de infinidad de obras, que repercutieron en la producción y comercialización a nivel internacional.

5. El cine alumbró unas obras brillantes enmarcadas en los géneros, consideradas como las primeras clásicas del cine, a veces a pesar de la censura.

6. La respuesta sindical fue en la dirección de colaborar para mantener en auge la actividad, casi lejos de la tradicional reivindicación obrera.

7. El nuevo programa reformista del New Deal se congració con la industria del cine, como revulsivo sociológico de esperanza y ánimo.

Son cuestiones que se retroalimentaron inexorablemente, dando a conocer la realidad de los comienzos del cine más universal e imperecedero. Resultaron unos condicionantes de gran relevancia, que llegaron a superar con holgura los tremendos vaivenes socioeconómicos de aquella época incierta. 
Hernández Rubio, J. Particularidades y contradicciones del cine norteamericano en los inicios del periodo clásico

\section{REFERENCIAS BIBLIOGRÁFICAS}

Allen, R.C. y Gómery, D. (1985). Teoría y práctica de la historia del cine. Barcelona: Paidós.

Bordwell, D., Thompson, K. y Staiger, J. (1997). El cine clásico de Hollywood: estilo cinematográfico y modo de producción hasta 1960. Barcelona: Paidós.

Coursodon, J.P. (1996). La evolución de los géneros. En VV.AA, Historia General del Cine. Estados Unidos (1932-1955) Vol. VIII (p.231). Madrid: Cátedra.

Cavell, S. (1999). La búsqueda de la felicidad. La comedia de enredo matrimonial en Hollywood. Barcelona: Paidós.

Davidson, D. (1993). Depression America and the Rise of the Social Documentary Film Author. In Published Chicago Review Stable, 34 (1) 6988.

Epstein, E.J. (2007). La gran ilusión. Dinero y poder en Hollywood. Barcelona: Tusquets.

Galbraith, J.K. (2000). El crac del 29. 5 a ed. Barcelona: Ariel.

Hueso, A. L. (1988). El cine y el siglo XX. Barcelona: Ariel.

Ibars, R. y López, I. (2006). La historia y el cine. Revista Clio, 32, 11. Recuperado de http://clio.rediris.es/n32/historiaycine/historiaycine.htm

Lacolla, E. (2008). El cine en su época. Una historia política del film. Córdoba (Argentina): Comunicarte.

Mainer, C. (2013). El cine norteamericano durante la Gran Depresión (19291939). Fotocinema. Revista científica de cine y fotografía, 6, 171-200.

Recuperado de http://www.revistafotocinema.com/index.php?journal=fotocinema\&page=arti cle\&op=view\&path $\% 5 \mathrm{~B} \% 5 \mathrm{D}=153$.

Marias, M. (1994). William Wellman, un cineasta misterioso. Revista Filmoteca Generalitat Valenciana, 146, 44.

Monterde, J.E. (1994). Hollywood en silencio. La llegada del sonoro. Revista Dirigido por, 116, 17.

Monterde, J.E. (1997). La imagen negada: Representación de la clase trabajadora en el cine. Valencia: Filmoteca de la Generalitat Valenciana.

Muscio, G. (1996). El cine americano de los años 30. En VV.AA, Historia General del Cine. Estados Unidos (1932- 1955) Vol. VIII (p.25). Madrid: Cátedra. 
Hernández Rubio, J. Particularidades y contradicciones del cine norteamericano en los inicios del periodo clásico

Muscio, G. (2011). Cine: producción y modelos sociales y culturales en los años treinta. En VV.AA. Historia Mundial del cine. Estados Unidos (p. 503). Madrid: Akal

Roffman, P. y Purdy, J. (1981). The Hollywood Social Problem Film: Madness, Despair and Politics from the Depression to the fifties. Bloomington: Indiana University Press.

Sadoul, V. (1991). Historia del Cine Mundial. México D.F.-Madrid: Siglo XXI.

Schwartz, P. (2009). La Gran Depresión de 1929 a 1940. Anales de la Real Academia de Ciencias Morales y Políticas, 86, 455-467. Recuperado de http://www.racmyp.es/R/racmyp/docs/anales/A86/A86-25.pdf

Staiger, J. (1995). Un cambio en la estructura de gestión del modo de producción. En VV.AA, Historia General del Cine. La transición del mudo al sonoro Vol. VI (pp. 109-145). Madrid: Cátedra.

Truslow, J.A. (1931). El nacimiento del sueño americano. Recuperado de: http://www.curistoria.com/2017/01/el-nacimiento-del-sueno-americano.html.

\section{AUTOR}

\section{José Hernández Rubio}

Doctor en Humanidades por la Universidad Carlos III de Madrid (2014). Máster en Historia y Estética de la Cinematografía, Universidad Valladolid (2008-2010) y Máster Investigación y Gestión del Patrimonio Histórico, Artístico y Cultural, Universidad de Murcia (2009). Docente en la Universidad del Mar (Universidad Murcia): "Educación en Valores Humanos en el aula a través del Cine" y "El cine como recurso didáctico en la enseñanza de las Humanidades" y en la Universidad Autónoma de Zacatecas (México): "Aprendizaje de valores democráticos, análisis político y derechos humanos a través del cine. Autor de libros científicos y ponente en diversos Congresos Internacionales tales como el I Congreso Internacional Historia, Literatura y Arte en el Cine en Español y en Portugués (Salamanca, 2011) o el V Congreso Internacional Historia y Cine (Uc3m, 2016).

http://orcid.org/0000-0001-7505-6613 